\title{
What are the current limits on determination of protonation state using neutron $\mathrm{MX}$ ?
}

\author{
D. Liebschner ${ }^{1}$, P. Afonine ${ }^{2}$, N. Moriarty ${ }^{3}$, P. Adams ${ }^{4}$ \\ ${ }^{1}$ Lawrence Berkeley National Laboratory ${ }^{2}$ No affiliation given, ${ }^{3}$ Lawrence Berkeley National \\ Laboratory, ${ }^{4}$ Lawrence Berkeley Laboratory \\ dcliebschner@gmail.com
}

The rate of deposition of models determined by neutron diffraction has increased in recent years. The benefit of neutron diffraction is that hydrogen atom positions are detectable. This allows determining protonation state and water molecule orientation. We analysed all neutron models deposited in the Protein Data Bank to date, focusing on protonation state and properties of hydrogen $(\mathrm{H})$ or deuterium (D) atoms as well as the details of water molecules. In particular, clashes and hydrogen bonds involving $\mathrm{H}$ or $\mathrm{D}$ atoms were investigated. As water molecules are typically the least reproducible part of a structural model, their positions in neutron models were compared to those in homologous high-resolution X-ray structures. For models determined by joint refinement against X-ray and neutron data, the water structure comparison was also carried out for models re-refined against the X-ray data alone (Fig. 1). The homologues have generally fewer conserved water molecules where X-ray only was used and the positions of equivalent waters vary more than in the case of the hybrid X-ray model. As neutron diffraction data are generally less complete than X-ray data, the influence of neutron data completeness on nuclear density maps was also analyzed. We observe and discuss systematic map quality deterioration as result of data incompleteness.

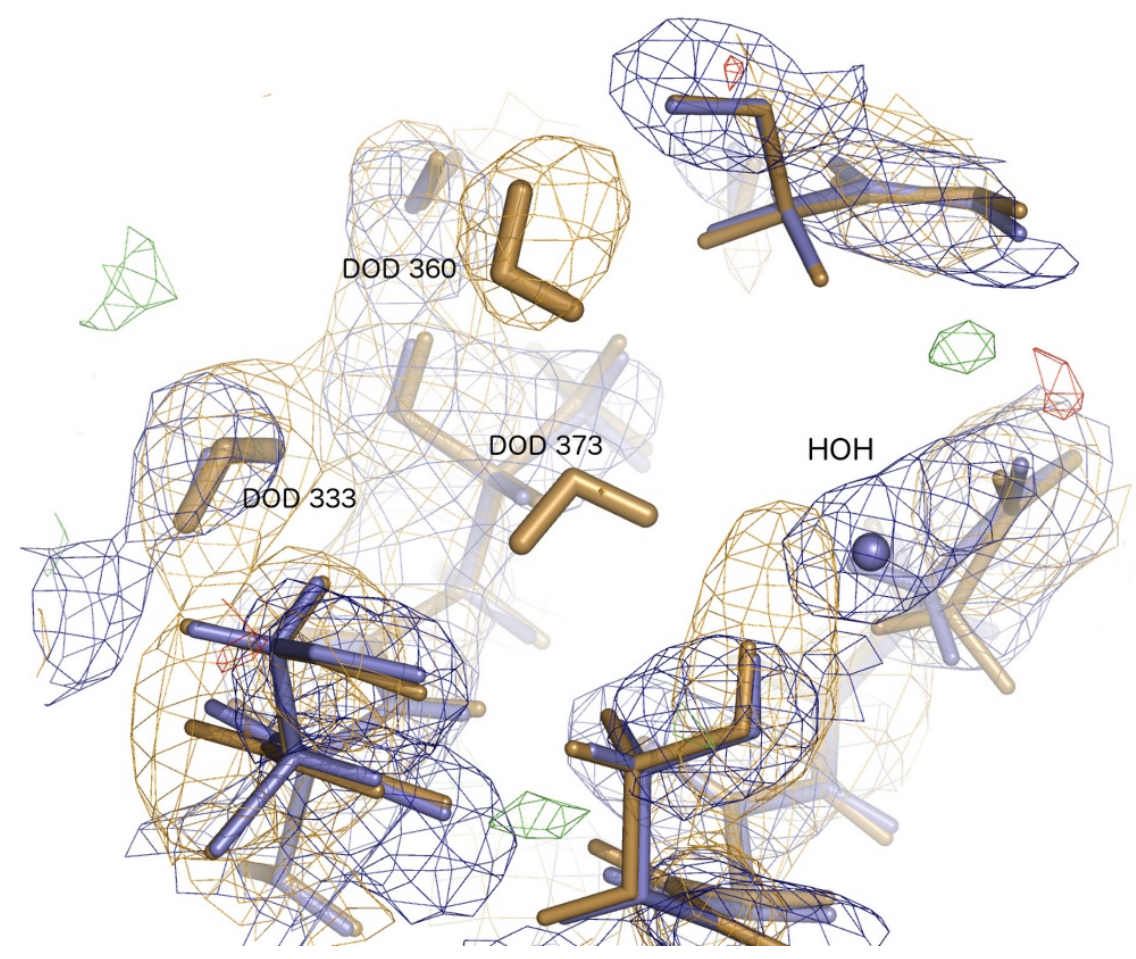

Figure 1 